Der Swiss Quality Award steht für Engagement im Gesundheitswesen. Er zeichnet Ideen aus, die neue Akzente im Qualitätsmanagement setzen. Im Zentrum stehen Pionierinnen und Pioniere, die mit viel Kreativität und noch mehr Engagement neue Ideen entwickeln und umsetzen, um die Qualität im Gesundheitswesen voranzubringen. Mit der Preisverleihung möchten die drei Trägerorganisationen SQMH (Schweizerische Gesellschaft für Qualitätsmanagement im Gesundheitswesen), IEFM (Institut für Evaluative Forschung in der Medizin der Universität Bern) und die FMH innovative und praxisorientierte Projekte einem grösseren Publikum zugänglich machen. Sie wollen damit aktiv die Vernetzung und die Weiterführung von wertvollen Qualitätsprojekten fördern.
Am 15. Mai 2012 war es wieder so weit: Aus knapp 60 eingereichten Projekten wurden vier zukunftsweisende und praxisnahe Arbeiten, welche die Qualität unseres Gesundheitswesens mit ihrem Engagement verbessern möchten, mit dem Swiss Quality Award ausgezeichnet. Gerne präsentieren die drei Trägerorganisationen die vier Siegerprojekte in der Schweizerischen Ärztezeitung. In dieser Ausgabe stellen wir Ihnen die Gewinner der Kategorien «Patientensicherheit» und «Empowerment» vor. Mehr über die Siegerprojekte der Kategorien «Management» und «Technologie» erfahren Sie im kommenden Heft der Schweizerischen Ärztezeitung.

Dr. med. Daniel Herren MHA, Mitglied des Zentralvorstandes der FMH, Verantwortlicher für das Ressort DDQ (im Amt bis 7.6.2012)

\title{
Swiss Quality Award 2012 - die Gewinner (Teil 1)
}

\section{Gewinner Kategorie Patientensicherheit}

\section{Jens Fischer, Adrian Steiner}

Spital Oberengadin, Institut f. Diagnostische u. Interventionelle Radiologie, Samedan
Korrespondenz:

Dr. med. Jens Fischer Spital Oberengadin Institut für Diagnostische Radiologie Via nuova 3

CH-7503 Samedan

Tel. 0818518614

fischer.jens[at]spital.net

\section{Optimierte systematische Dosisreduktion in der Computertomographie mittels iterativer Rekonstruktion unter Berücksichtigung des BMI}

Im Jahr 2008 lag die Schweiz bei der kollektiven Strahlenbelastung pro Kopf, verursacht durch die 20 häufigsten radiologischen Untersuchungen, in Europa hinter Deutschland und Belgien auf Platz drei [1]. Die Strahlenbelastung, verursacht durch Computertomographien, hat hierbei jeweils den grössten Anteil. Die Zahl jährlicher CT-Untersuchungen ist zudem weltweit am Steigen [2].

Um die Strahlenbelastung durch CT-Untersuchungen zu minimieren, wurden von der Industrie zahlreiche Systeme zur Strahlendosis-Einsparung entwickelt. Die neueste dieser Entwicklungen ist die Anwendung der «iterativen Rekonstruktion», bei der eine Bildoptimierung durch aufwendige, nachbearbeitende Rechenprozesse erreicht wird [3]. Diese sekundäre Bildoptimierung macht es möglich, deutlich geringere Strahlendosen unter Beibehaltung der Bildqualität zu verwenden. Der Anwender steht jedoch auch vor der Herausforderung, diese Methode im radiologischen Routinealltag so optimal wie möglich zu nutzen bzw. einzusetzen.

Ziel der Autoren war es, ein möglichst einfaches und somit im klinischen Alltag leicht zu integrierendes System zu entwickeln, das die iterative Rekonstruktion bestmöglich nutzt. Dieses System sollte den Körperhabitus bzw. die erforderliche Exposition so optimal wie möglich berücksichtigen und zudem eine der Fragestellung genügende bzw. diagnostische Bildqualität liefern.

Zu diesem Zweck wurden 4 definierten BMI-Klassen (S, M, L, XL) entsprechende dosisreduzierte CTProtokolle (Thorax und Abdomen) inkl. der Stufe der iterativen Rekonstruktion zugeordnet und im Gerät hinterlegt. Diese Protokolle wurden im Verlauf immer wieder unter Berücksichtigung des «ALARA»Prinzips (As Low As Reasonable Achievable) angepasst. Durch die Einführung des beschriebenen Systems liessen sich im Spital Oberengadin innerhalb kurzer Zeit zwei wesentliche Ergebnisse erzielen:

- Senkung der durchschnittlichen Dosiswerte für Thorax-/Abdomen-Untersuchungen in den Bereich der 25. Perzentile der in der Schweiz gemessenen Dosisverteilung (gemäss BAG Merkblatt R-06-06).

\section{Swiss Quality Award - Innovations in Healthcare}

Der Swiss Quality Award wird in den vier Kategorien Management, Patientensicherheit, Technologie und Empowerment verliehen. Getragen wird der Preis von drei Organisationen: der Schweizerischen Gesellschaft für Qualitätsmanagement im Gesundheitswesen (SQMH), dem Institut für Evaluative Forschung in der Medizin (IEFM) der Universität Bern sowie der Verbindung der Schweizer Ärztinnen und Ärzte (FMH). Alle Informationen zum Swiss Quality Award sowie eine Onlineplattform mit den Projektpostern der Gewinner und weiteren Projekten befinden sich unter www.swissqualityaward.ch 


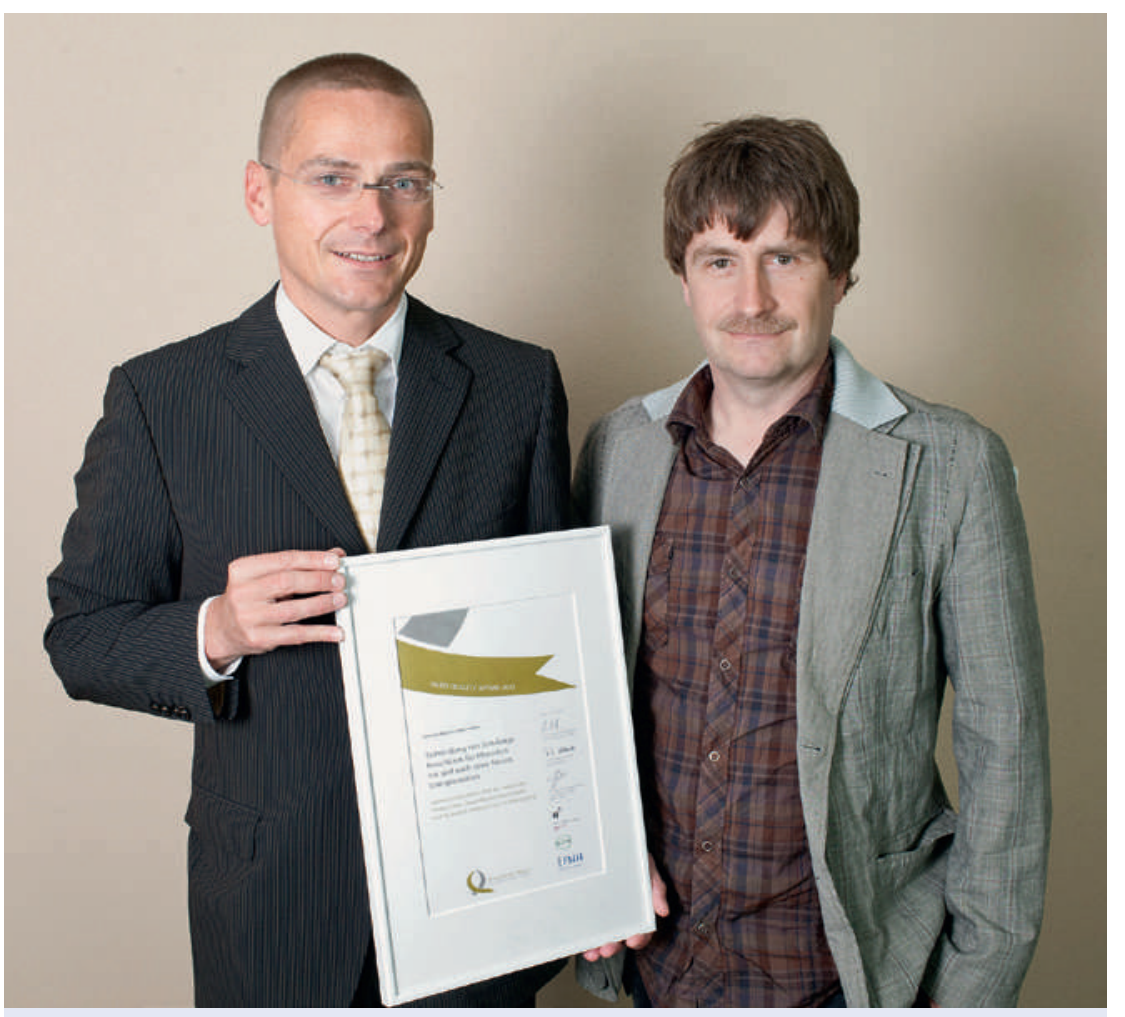

Jens Fischer und Adrian Steiner: Gewinner des Swiss Quality Awards 2012 in der Kategorie Patientensicherheit.
- Schnelle Integration dieses Dosisreduktionssystems in den radiologischen Routinebetrieb, ohne relevanten zeitlichen oder personellen Mehraufwand.

Die Einführung dieser systematischen und einfach durchführbaren CT-Dosisreduktion führte im Spital Oberengadin zu einer signifikanten und nachhaltigen, weit über die Zielvorgaben des BAG hinausgehenden Reduktion der Strahlenexposition. Die Autoren sind überzeugt, dass hierdurch eine signifikante Reduktion der Strahlenbelastung der Gesamtpopulation möglich ist und somit ein elementarer Beitrag zur Patientensicherheit geleistet werden kann.

Die Autoren planen, dieses System hinsichtlich Untersuchungsart, individueller Dosisoptimierung, Bildqualität sowie Automatisierung auch in Zukunft weiter auszubauen bzw. zu verbessern.

\section{Literatur}

1 Aroua A et al. in: Rautakoski T, Heron L, Donald L, Jagt VD. Session 2: Medical use of radiation. Oral presentations. Ariadne; 2010.

2 Hart D, Wall BF, Hillier MC, Shrimpton PC. Frequency and Collective Dose for Medical and Dental X-ray Examinations in the UK; 2008.

3 Hara AK, Paden RG, Silva AC, et al. Iterative reconstruction technique for reducing body radiation dose at CT: feasibility study. AJR. American journal of roentgenology. 2009;193(3):764-71.

\section{Gewinner Kategorie Empowerment}

\section{G. Schmid-Mohler ${ }^{a, b}$,}

T. Fehr ${ }^{a}$, T. Albiez ${ }^{c}$, P.

Witschi ${ }^{a}$, B. Biottic,

R. P. Wüthrich ${ }^{a}$, R. Spirig b,d

a Klinik für Nephrologie, UniversitätsSpital Zürich

b Zentrum Klinische Pflegewissenschaft,

UniversitätsSpital Zürich

c Medizinbereich Abdomen Stoffwechsel,

UniversitätsSpital Zürich

d Institut für Pflegewissenschaft, Universität Basel

Korrespondenz:

Gabriela Schmid-Mohler UniversitätsSpital Zürich Zentrum für klinische Pflegewissenschaft,

Rämistrasse 100

CH-8091 Zürich

Tel. 0442552003

gabriela.schmid[at]usz.ch

\section{Schulungsbroschüren für Menschen vor und nach einer Nierentransplantation}

Nierentransplantierte Menschen müssen sich neues Wissen und Fähigkeiten aneignen, um ihr Leben nach der Transplantation zu meistern. Eine Analyse der Versorgungspraxis von Nierenempfängern im UniversitätsSpital Zürich (USZ) auf der Basis des Chronic Care Modell der WHO [1] mit qualitativen und quantitativen Methoden zeigte, dass die Betroffenen in ihrem Selbstmanagement wenig systematisch und nicht individuell genug unterstützt wurden. Informationen zu psychosozialen und präventiven Themen fehlten ebenso wie evidenzbasierte Richtlinien und Schulungsmaterial zu gesundheitsrelevantem Verhalten. Vor diesem Hintergrund wurde das «Selbstmanagementprogramm vor und nach Nierentransplantation» initiiert. Das erste Ziel des Programms war, evidenzbasierte Schulungsbroschüren zu erarbeiten.

Die Themen der Broschüren wurden ausgewählt aufgrund der aktuellen Literatur und der Sichtweisen von nierentransplantierten Menschen, Patientenorganisationen und der im Patientenpfad involvierten Berufsgruppen. Die Inhalte wurden dann unter Einbezug von Experten und einer nierentransplantierten Person erarbeitet. $\mathrm{Zu}$ definierten Themen wurde systematisch Literatur gesucht, beurteilt und

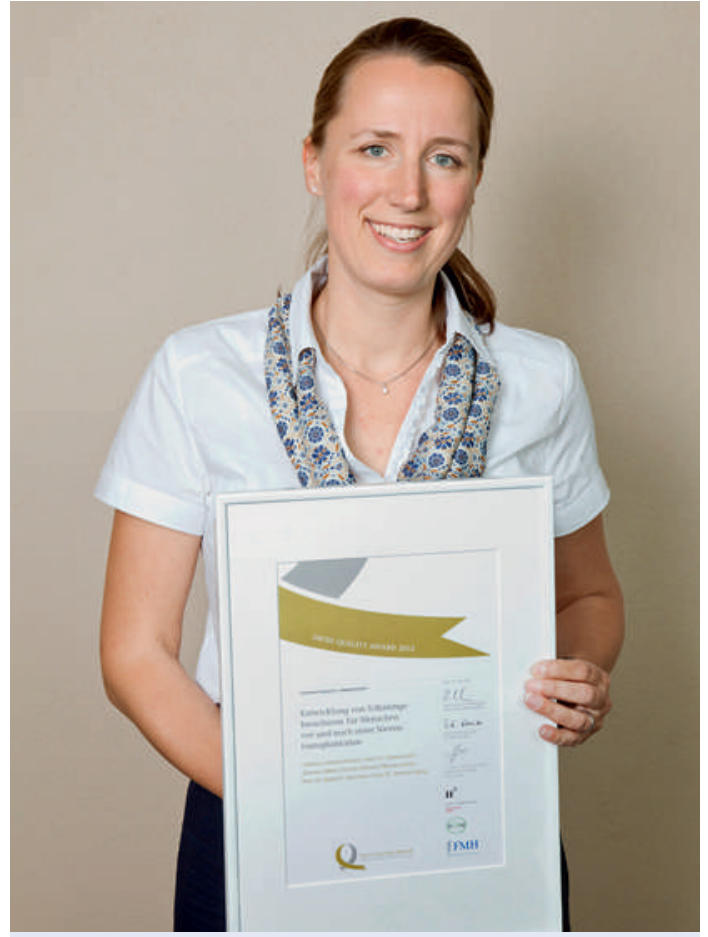

Gabriela Schmid-Mohler: Vertreterin des Gewinnerteams der Kategorie «Empowerment» des Swiss Quality Awards 2012. 
eingearbeitet. Empfehlungen wurden in Absprache mit den jeweiligen Experten formuliert.

Personen vor oder kurz nach einer Nierentransplantation sowie verschiedene Fachpersonen aus der Pflege und der Ärzteschaft beurteilten den Entwurf anhand folgender Kriterien: 1. Relevanz und Vollständigkeit des Inhaltes, 2. Einbezug der Prozessvariablen des Selbstmanagements, basierend auf dem ausgewählten theoretischen Rahmen [2], 3. Einarbeitung der Evidenz, 4. Verständlichkeit, 5. Übersichtlichkeit 6. Einhalten von formalen Aspekten und 7. Allgemeiner Eindruck. Die Evaluation ergab, dass die Broschüren die Kriterien überwiegend oder völlig erfüllten. Der Entwurf wurde aufgrund der Evaluation überarbeitet.

Das Ergebnis besteht aus drei Broschüren. Während sich die erste Broschüre auf die Zeit vor der Nierentransplantation bezieht, befasst sich die zweite mit Themen der Akutphase und die dritte mit Themen des Langzeitmanagements. Alle Broschüren legen den Fokus auf die Förderung gesunden Verhaltens. Sie geben konkrete Empfehlungen ab und beziehen sich auf den Alltag. Neben medizinischen Themen behandeln sie wichtige psychosoziale Fragen, beispielsweise den Umgang mit Niedergeschlagenheit oder die Sorge, die Niere zu verlieren. Jede Broschüre enthält spezifische Kriterien für den Umgang mit Symptomen und Situationen. Dies ermöglicht betroffenen Personen, sich darüber zu orientieren, wie weit sie sich selber versorgen können. Sie erhalten Entscheidungshilfen, wann es sinnvoll und unabdingbar ist, eine Fachperson aufzusuchen. Ferner bieten die Broschüren Erfahrungsberichte von

\section{Ausschreibung 2013}

Nach der Preisverleihung ist vor der Preisverleihung! Der Swiss Quality Award wird auch 2013 erneut verliehen. Neben neuen Projekten sind auch Projekte willkommen, die sich schon mal um den Swiss Quality Award beworben haben. Bedingung ist jedoch, dass sich ein Projekt deutlich weiterentwickelt hat und diese Entwicklung auch dokumentiert wird. Die Preisausschreibung ist für den Herbst 2012 geplant.

Betroffenen. Diese zeigen auf, wie Menschen transplantationsbedingte Herausforderungen gemeistert haben.

Die Broschüren werden innerhalb des Betreuungsprozesses abgegeben. Die Abgabe der Broschüren 2 und 3 ist mit Schulungsgesprächen verknüpft. Das Selbstmanagementprogramm wird weiterentwickelt mit dem Ziel, nierentransplantierte Personen zu motivieren und zu befähigen, ihr Wissen und Verhalten eigenständig und nachhaltig im persönlichen Alltag umzusetzen.

Die Entwicklung wurde durch Novartis Pharma AG Schweiz unterstützt.

\section{Literatur}

1 Epping-Jordan JE et al., Improving the quality of health care for chronic conditions. Qual Saf Health Care. 2004;13(4):299-305.

2 Lorig KR, Holman H. Self-management education: history, definition, outcomes, and mechanisms. Ann Behav Med. 2003;26(1):1-7. 